\title{
Investigation of Methacrylic Acid at High Pressure using Neutron Diffraction.
}

\author{
William G. Marshall, ${ }^{\mathrm{b}}$ Andrew J. Urquhart ${ }^{\mathrm{c}}$ and Iain D.H. Oswald. ${ }^{\mathrm{a}^{*}}$
}

${ }^{a}$ Strathclyde Institute of Pharmacy and Biomedical Sciences, University of Strathclyde, 161 Cathedral Street, Glasgow, UK, G4 0RE. Tel: +441415482157. Email: iain.oswald@ strath.ac.uk

${ }^{\mathrm{b}}$ ISIS Neutron and Muon Source, Science and Technology Facilities Council, Rutherford Appleton Laboratory, Harwell Oxford, Didcot, Oxon, UK OX11 0QX.

${ }^{\mathrm{c}}$ Department of Micro- and Nanotechnology, Building 345Ø, Ørsteds Plads, Technical University of Denmark, 2800 Kgs. Lyngby, Denmark.

\begin{abstract}
This article shows that pressure can be a low intensity route to the synthesis of polymethacrylic acid. The exploration of perdeuterated methacrylic acid at high pressure using neutron diffraction reveals that methacrylic acid exhibits two polymorphic phase transformations at relatively low pressures. The first is observed at $0.39 \mathrm{GPa}$ where both phases were observed simultaneously and confirm our previous observations. This transition is followed by a second transition at $1.3 \mathrm{GPa}$ to a new polymorph that is characterised for the first time. On increasing pressure the diffraction pattern of phase III deteriorates significantly. On decompression phase III persists to $0.54 \mathrm{GPa}$ before transformation to the ambient pressure phase. There is significant loss of signal after decompression signifying that there has been a loss of material through polymerisation. The orientation of the molecules in Phase III provides insight into the possible polymerisation reaction.
\end{abstract}

\section{Introduction}

High pressure has been successfully used by a number of groups to create polymers from monomeric species. These solid-state reactions have employed pressure alone or laser irradiation under high pressure to achieve polymerisation. ${ }^{1}$ Polymorphism in these systems is critical to the outcome of these reactions as the proximity of reaction centres in the crystal structure (e.g. unsaturated bonds) will play a role in a) the ability of the polymerisation to occur and b) the architecture or tacticity of the resulting polymer backbone. Systems that have been investigated include ethylene ${ }^{2}$, benzene ${ }^{3}$, co-crystalline materials such as oxalamides and diiodobutadiyne ${ }^{4-5}$ and simple systems such as $\mathrm{CO}_{2}{ }^{6}$ Further work has 
extended the investigation of high-pressure polymerisation to ring systems such as L,Llactide $^{7}$ and carnosine. ${ }^{8}$

In this article, we continue our structural studies into the behaviour of small molecule monomers by investigating the room-temperature behaviour of methacrylic acid (Scheme 1) at high pressure using neutron diffraction. Methacrylic acid is a methylated derivative of acrylic acid which we have previously studied. ${ }^{9-10}$ It is used as a precursor to methyl methacrylate which forms polymethylmethacrylate (PMMA), one of the most widely used thermoplastics in the world. Our interest in methacrylic acid is to observe the effects that the addition of a methyl group close to the reactive moiety will have on the ability of the compound to polymerise under pressure especially as steric effects become more important as volume is reduced. ${ }^{11}$ We have previously investigated the structure of methacrylic acid- $h_{6}$ under low temperature and high pressure conditions where we observed two different crystal forms depending on the experimental conditions. Crystallisation of methacrylic acid via cooling below its melting point of $287 \mathrm{~K}$ yielded one polymorph (Phase I). A second form (Phase II) was isolated at high pressure $(0.64 \mathrm{GPa}$ with data collected at $1.5 \mathrm{GPa})$ however some initial neutron diffraction experiments suggested that further phases of methacrylic acid existed that had not previously been isolated. Neutron diffraction is very useful for these types of systems as our previous study of acrylic acid revealed that X-ray radiation from the single crystal X-ray diffraction experiment may have started to cause the polymerisation; neutrons did not have the same effect in acrylic acid allowing for a truer reflection of structural changes occurring with respect to pressure as opposed to the probing radiation. ${ }^{9-10}$ In fact, syndiotactic polymethacrylic acid was created by irradiating a methacrylic acid solution in 1-propanol with 10 Mrad of cobalt-60 $\gamma$-radiation therefore pressure may be an avenue to create polymers of monomeric complexes that are difficult to create by ambient pressure methods. ${ }^{12}$ Herein we present our work on the structural changes that occur in methacrylic acid with respect to pressure and the subsequent polymerisation.

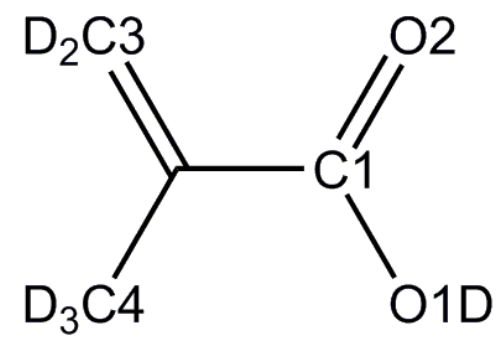

Scheme 1: Numbering scheme for methacrylic acid- $d_{6}$ 


\section{Experimental Section}

\section{Neutron Diffraction measurements}

High-pressure neutron powder diffraction data were collected for methacrylic acid- $d_{6}$ using the PEARL diffractometer ${ }^{13-14}$ at the UK spallation neutron source, ISIS, located at the STFC Rutherford Appleton Laboratory. The procedures for the loading of the V3 Paris-Edinburgh press ${ }^{15}$ in the neutron experiment follow those in our previous paper on acrylic acid- $d_{4}$ and references therein. ${ }^{10}$ For this experiment zirconia-toughened alumina anvils were used. ${ }^{16}$ The sample was first mixed with $\sim 20 \%$ 4:1 methanol- $d_{4}$ :ethanol- $d_{6}$ before being added dropwise using a glass capillary into a standard encapsulated nullscattering Ti-Zr alloy gasket ${ }^{17}$ filled with loosely-packed ground silica wool, which was used to inhibit the formation of large crystallites, and calcium fluoride as a pressure marker. ${ }^{18}$ The methanol:ethanol mixture was used as a pressure-transmitting medium (PTM) to provide quasi-hydrostatic conditions during the compression. Two loadings were conducted on two separate visits to ISIS. The Time-of-flight (TOF) neutron powder diffraction data were collected as per our previous work and analysed with Topas Academic using a Z-matrix model parameterised in terms of the intramolecular bond distances, angles and torsions and the molecular position and orientation. ${ }^{19}$ The starting models were derived from our previous X-ray diffraction study. ${ }^{9}$ The DFT-optimised structures (see below) were used to formulate restraints which were then applied to the Rietveld refinements as described in ref ${ }^{20}$.

Table 1: Crystallographic information for the lowest and highest pressure Rietveld refinements for Phase I, Phase II and Phase III of methacrylic acid- $d_{6}$.

\begin{tabular}{|l|c|c|c|c|}
\hline CIF ID & 1 & 3 & 4 & 10 \\
\hline Pressure $(\mathrm{GPa})$ & $0.124(6)$ & $0.395(8)$ & $0.395(8)$ & $1.296(8)$ \\
\hline Chemical formula & $\mathrm{C}_{4} \mathrm{H}_{6} \mathrm{O}_{2}$ & $\mathrm{C}_{4} \mathrm{H}_{6} \mathrm{O}_{2}$ & $\mathrm{C}_{4} \mathrm{H}_{6} \mathrm{O}_{2}$ & $\mathrm{C}_{4} \mathrm{H}_{6} \mathrm{O}_{2}$ \\
\hline$M_{r}$ & 86.06 & 86.06 & 86.06 & 86.06 \\
\hline Radiation type & Neutron & Neutron & Neutron & Neutron \\
\hline Phase & I & I & II & II \\
\hline $\begin{array}{l}\text { Cell setting, space } \\
\text { group }\end{array}$ & Monoclinic, $P 2_{1} / c$ & Monoclinic, $P 2_{1} / c$ & Monoclinic, $P 2_{1} / c$ & Monoclinic, $P 2_{1} / c$ \\
\hline Temperature $(\mathrm{K})$ & 293 & & & 293 \\
\hline $\mathrm{a}(\AA)$ & $3.8820(2)$ & 293 & 293 & $3.7760(19)$ \\
\hline $\mathrm{b}(\AA)$ & $10.6085(16)$ & $10.452(4)$ & $13.704(2)$ & $13.481(7)$ \\
\hline $\mathrm{c}(\AA)$ & $11.7661(17)$ & $11.598(4)$ & $8.6087(11)$ & $8.352(5)$ \\
\hline$\beta\left({ }^{\circ}\right)$ & $91.297(8)$ & $89.93(3)$ & $102.680(10)$ & $101.43(4)$ \\
\hline
\end{tabular}




\begin{tabular}{|l|c|c|c|c|}
\hline Volume $\left(\AA^{3}\right)$ & $484.43(10)$ & $459.8(3)$ & $453.18(10)$ & $416.7(4)$ \\
\hline $\mathrm{D}\left(\mathrm{g} \mathrm{cm}^{-1}\right)$ & 1.180 & 1.244 & 1.262 & 1.372 \\
\hline $\mathrm{Z}$ & 4 & 4 & 4 & 4 \\
\hline $\mathrm{R}_{\mathrm{wp}}$ & 4.018 & 6.242 & 6.242 & 5.520 \\
\hline \hline CIF ID & 12 & 19 & $1-\mathrm{SXD}$ & \\
\hline Pressure $(\mathrm{GPa})$ & $1.296(8)$ & $3.891(12)$ & & \\
\hline Chemical formula & $\mathrm{C}_{4} \mathrm{H}_{6} \mathrm{O}_{2}$ & $\mathrm{C}_{4} \mathrm{H}_{6} \mathrm{O}_{2}$ & $\mathrm{C}_{4} \mathrm{H}_{6} \mathrm{O}_{2}$ & \\
\hline$M_{r}$ & 86.06 & 86.06 & 86.06 & \\
\hline Radiation type & Neutron & Neutron & X-ray, Mo-Ka & \\
\hline Phase & III & III & III & \\
\hline $\begin{array}{l}\text { Cell setting, space } \\
\text { group }\end{array}$ & Monoclinic, $P 2_{1} / n$ & Monoclinic, $P 2_{1} / n$ & Monoclinic, $P 2_{1} / n$ & \\
\hline Temperature $(\mathrm{K})$ & & & & \\
\hline $\mathrm{a}(\AA)$ & 293 & 293 & 293 & \\
\hline $\mathrm{b}(\AA)$ & $3.6702(4)$ & $3.4531(12)$ & $3.6140(7)$ & \\
\hline $\mathrm{c}(\AA)$ & $16.498(3)$ & $16.124(11)$ & $16.420(3)$ & \\
\hline$\beta\left({ }^{\circ}\right)$ & $6.8167(10)$ & $6.598(3)$ & $6.773(3)$ & \\
\hline Volume $\left(\AA^{3}\right)$ & $89.84(4)$ & $89.22(5)$ & $90.36(3)$ & \\
\hline $\mathrm{D}\left(\mathrm{g} \mathrm{cm}{ }^{-1}\right)$ & $412.77(11)$ & $367.3(3)$ & $401.91(17)$ & \\
\hline $\mathrm{Z}$ & 1.385 & 1.557 & 4.423 & \\
\hline $\mathrm{R}_{\mathrm{wp}}$ & 4 & 4.168 & $R\left[F^{2}>2 \sigma\left(F^{2}\right)\right]$, & \\
& 5.520 & & $w R\left(F^{2}\right), S$ & \\
\hline & & & & \\
\hline
\end{tabular}

Periodic DFT calculations

Geometry optimisations were performed by periodic Density Functional Theory (DFT) using the $\mathrm{DMOL}^{3}$ code $^{21}$ as part of the Materials Studio modelling suite. ${ }^{22}$ The DNP numerical basis $\operatorname{set}^{21}$ was used in combination with the PBE functional ${ }^{23}$ with the Tkatchenko- Scheffler correction for dispersion. ${ }^{24}$ The unit cell dimensions were held fixed at the values obtained in Pawley refinements of the neutron powder diffraction data described above, and coordinates were allowed to optimise. Convergence was defined when the maximum changes in total energy, displacement and gradient were $10^{-5} \mathrm{Ha}, 5 \times 10^{-3} \AA$ and 2 x $10^{-3} \mathrm{Ha} \AA^{-1}$, respectively. Brillouin zone integrations were performed by Monkhorst-Pack ${ }^{25}$ k-point sampling at intervals of $0.07 \AA^{-1}$.

\section{Pixel calculations}

Using the optimised structures from the DFT calculations, the lattice energies and molecule-molecule interaction energies were calculated using Pixelc module in the CLP package by Gavezotti. ${ }^{26-27}$ Electron densities were calculated at the MP2/6-31G** level using Gaussian09. ${ }^{28}$ For these calculations the deuterium atoms were substituted by hydrogen and normalized in Mercury $3.3^{29}$ before submission to Pixel. Table ES2 
provides the total lattice energy as well as the breakdown of intermolecular interactions into Coulombic, electrostatic, dispersion and repulsion terms.

\section{Results \& discussion}

\section{The effect of pressure on methacrylic acid- $d_{6}$}

One aspect of our previous work that was overlooked was the fact that the conformation of methacrylic acid changes whether it is crystallised at either low temperature or high pressure (same polymorph). At low temperature the molecule exists in the trans configuration, with respect to the ethylene and carbonyl moieties. Whereas if the same polymorph is recrystallised by applying pressure the molecule adopts the cis conformation; this is clearly indicated from the C-C bond lengths. Badawi et al. studied the conformational changes in methacrylic acid and observed that at various levels of theory (B3LYP/6-311G**, MP2/6-311G**) the energy difference between the cis and trans conformers was, at most, $\sim 3.5 \mathrm{~kJ} / \mathrm{mol}^{30}$ At both low temperature and under initial high-pressure conditions methacrylic acid- $d_{6}$ crystallises in Phase I, a monoclinic $P 2_{1} / c$ with a stepped layered structure (viewed down the $b$-axis) where the molecules interact through a carboxylic acid dimer interaction (Figure 1). As anticipated Pixel calculations show that the dimer interaction is the most favourable at $-60 \mathrm{~kJ} / \mathrm{mol}$ with all components, coulombic, polarisation and dispersion contributing significantly to the favourable nature of the interaction. Note that the energies are calculated based on molecule-molecule interactions and not just the dimer interaction hence there are other contributing features. The next two interactions (Int. 2 and 3) are similar in energy but the contributions made from each energetic term differs slightly (Figure 2). Interaction 2 is observed within a staggered layer where the methylene group is in close proximity to the carbonyl groups of the neighbouring dimer and the methyl group is situated close to the methylene group. This is reflected in the similar energetic contributions from the coulombic and dispersive terms. Interaction 3 is a 'face-to-face' interaction which leads to a large contribution from the dispersive term. The molecules are stacked so that the carbonyl interactions are in a parallel arrangement and are observed outside the 3.6 $\AA$ used as a cut-off by Allen et al. in their work on carbonyl groups. ${ }^{31}$ The longer interaction length can be attributed to the increased bulk of the methyl groups. The distribution of carbon-carbon distances in the Cambridge Structural Database is found in Figure ES1. ${ }^{31}$ This 'face-to-face' interaction in Phase I is significantly different from acrylic acid where the carbonyl groups interacted 
through an anti-parallel arrangement. ${ }^{10}$ The last interaction (Int. 4) is to the hydrogen bonded molecule in the layer above. This molecule is sufficiently far away to show little repulsion but contributions in the form of coulombic and dispersive components, presumably due to the proximity of the hydrogen bonding groups, make this a favourable interaction.

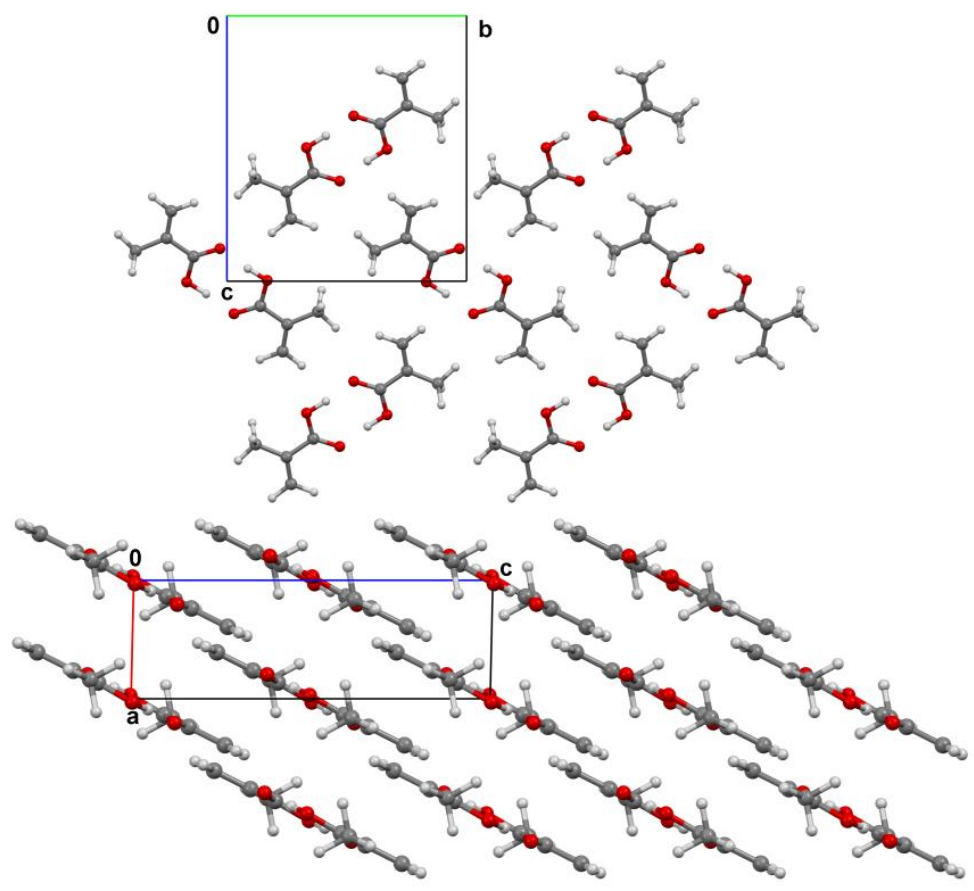

Figure 1: A layer of molecules observed in Phase I methacrylic acid showing the hydrogen bonded dimer (top). A view along the $b$-axis that shows the staggered layers of molecules (bottom). Atom colors are assigned as Grey, carbon; Red, oxygen; and White, hydrogen.

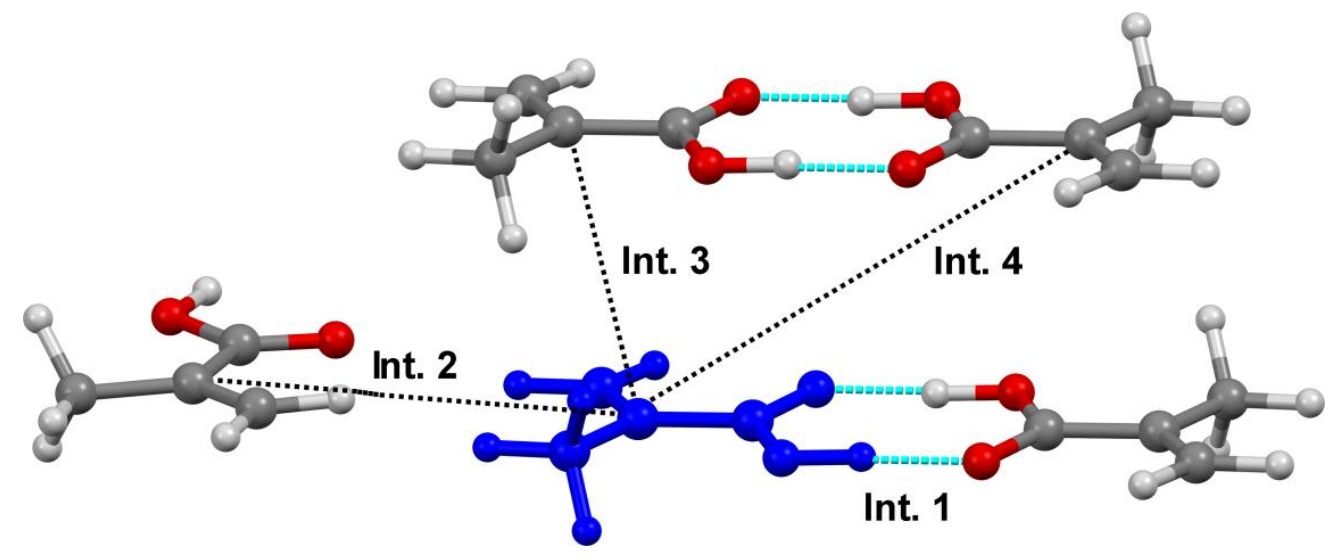

Figure 2: The most energetically favorable interactions in Form I of methacrylic acid with energies $>5$ $\mathrm{kJ} / \mathrm{mol}$ using the blue central molecule as a reference. Atom colors are assigned as Grey, carbon; Red, oxygen; and White, hydrogen. 
On compression to $0.39 \mathrm{GPa}$ the unit cell parameters of Phase I compress by $2.3 \%, 1.5 \%$ and $1.4 \%$ for the $a-, b-$, and $c$-axis, respectively, with the molecular volume reducing by $5 \%$ (Figure $3 \& 4$ ). This is a similar compression observed for acrylic acid to $0.69 \mathrm{GPa}$ $(4.47 \%) .{ }^{10}$ At $0.39 \mathrm{GPa}$ both Phase I and Phase II (identified from previous study at 0.64 $\mathrm{GPa}$ ) were refined against the data and showed that Phase II is $1.4 \%$ more dense. The III phase transition was quite slow and had not completed by the time we increased the pressure on the system (2hrs 40 mins; Figure ES2)); Figure ES3 shows the Rietveld fits for both phases. The intensity of the Phase I patterns suggests that it would have persisted for longer had we left it at this pressure but the kinetics of transformation were not a key outcome for the experiment. As soon as pressure was increased to $0.53 \mathrm{GPa}$ any reminence of Phase I had gone. Phase II is observed at $\sim 0.2 \mathrm{GPa}$ lower than our previous study. ${ }^{9}$ This can be accounted for by two factors i) the change in the ratio of PTM to acid used in this study. Here we have used 20\% PTM v/v as opposed to the 50\% $\mathrm{v} / \mathrm{v}$ used previously ${ }^{9}$ which has altered the pressure at which the methacrylic acid is precipitated out from solution; in this experiment it precipitated out at $0.12 \mathrm{GPa}$ whereas this occurred at $0.64 \mathrm{GPa}$ in our previous study and ii) the addition of the silica glass (for powder averaging) which provides nucleation points. 


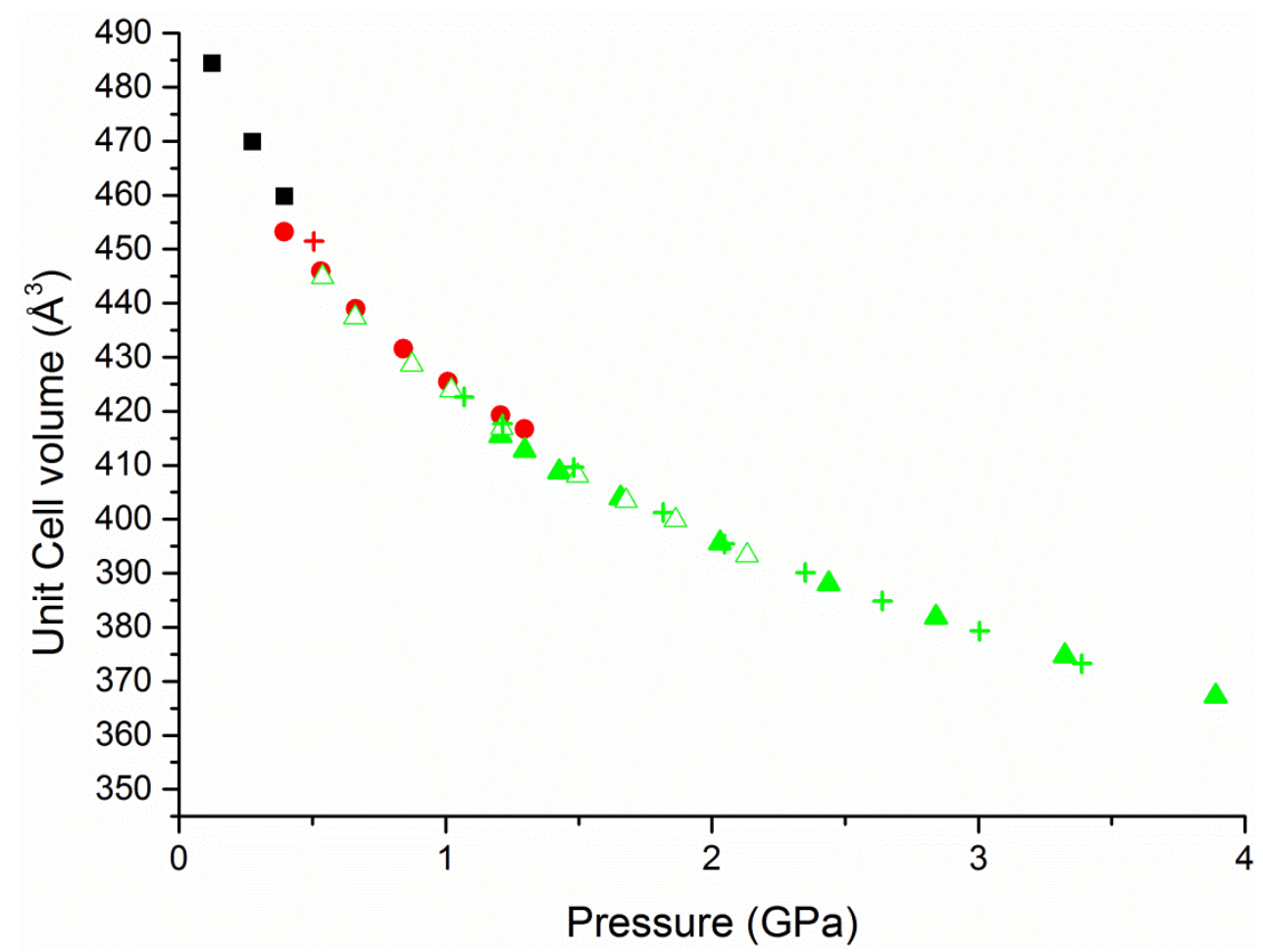

Figure 3: The unit cell volume as a function of pressure. The different phases are indicated by black squares (Phase I), red circles (Phase II) and green triangles (Phase III). The hollow triangles are unit cell parameters from decompression experiment. The crosses are data points from a second experiment conducted at a different beamtime allocation and largely fit with the first experiment.

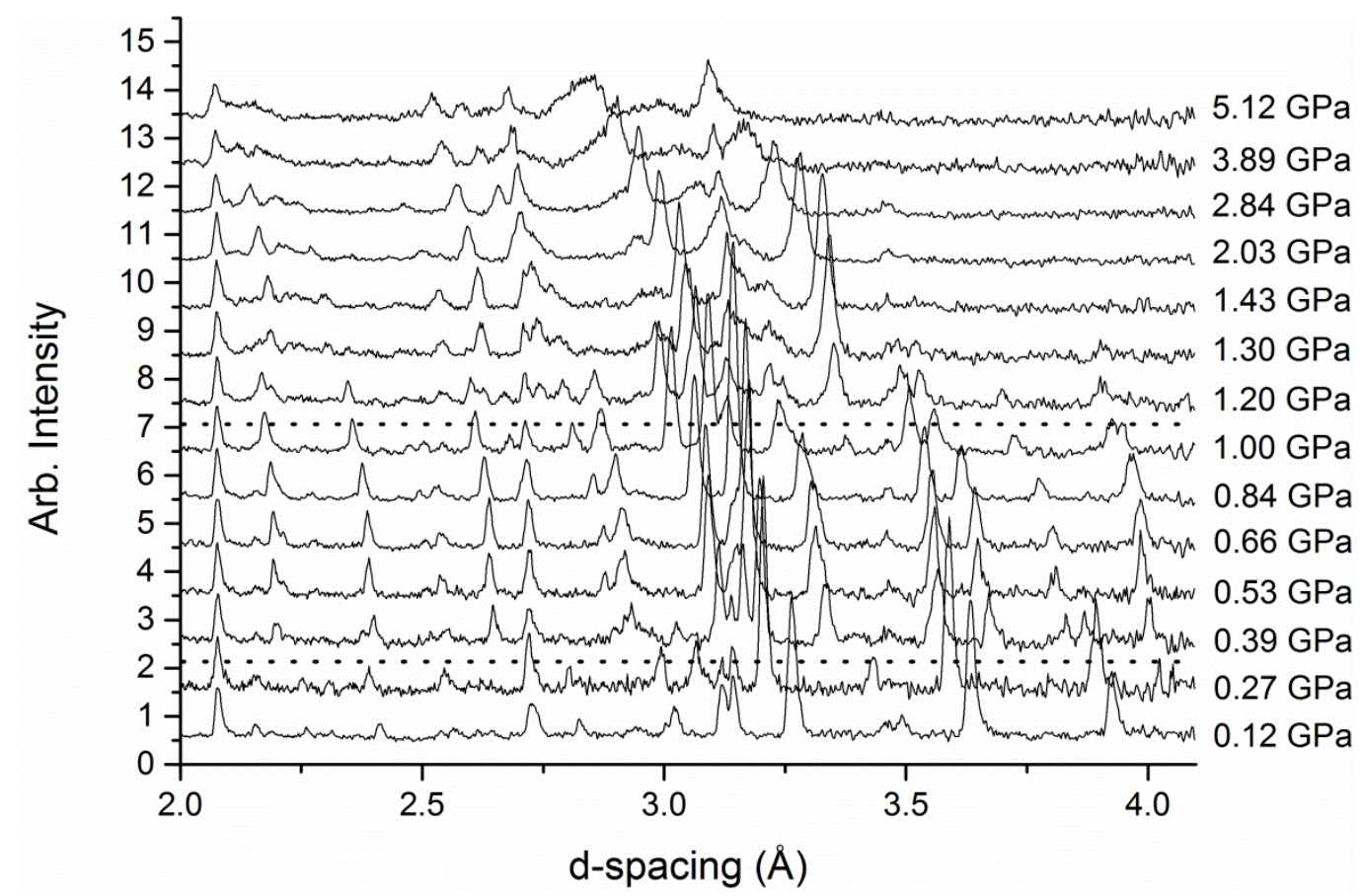

Figure 4: The diffraction patterns of methacrylic acid on increasing pressure. The horizontal dotted lines are indicative of where the phase boundaries are from Phase I-II (0.27-0.39 GPa) and Phase II-III (1.00$1.20 \mathrm{GPa})$. 
Phase II is a distinctly more layered structure than Phase I with an overall corrugated arrangement of molecules (Figure 5). The molecular conformation is approximately the same with an energy difference of $0.352 \mathrm{~kJ} / \mathrm{mol}$ from the conformation in Phase I. The spatial arrangement of molecules in the layer and between the layers sees a significant change from Phase I. As anticipated the interaction between the molecules is through the carboxylic acid dimer which is observed to be the strongest intermolecular interaction (Int. 1; O1... $22.57(2) \AA,-60 \mathrm{~kJ} / \mathrm{mol}$ ) but it is in the interaction between dimers that one observes a difference in the arrangement. In Phase I the interactions between the stacked layers in the plane is one of the more significant interactions (Int. 2, $-9 \mathrm{~kJ} / \mathrm{mol}$ ) the similar interaction in Phase II (Int. $5-6.2 \mathrm{~kJ} / \mathrm{mol}$ ) is rather less significant (Figure 6). This can be attributed to the shift laterally of the neighbouring dimer so that the methyl and methylene are not interacting as much with the oxygen atoms of the neighbouring molecule. This is reflected in the major energetic component being the dispersive interaction. Interaction 3 and 4 in both phases are similar to one another where the central molecule interacts with molecule in the layer above (or below) in the same stack. The separation between the least squares plane of the dimers is $3.51 \AA$ in Phase I versus 3.36 $\AA$ in Phase II but despite this the intermolecular energies are approximately similar. The dimers in Phase I are positioned so that the methyl groups are on top of one another whereas in Phase II the dimers have slipped with respect to one another alleviating any steric issues and allowing a closer spacing between dimers. 

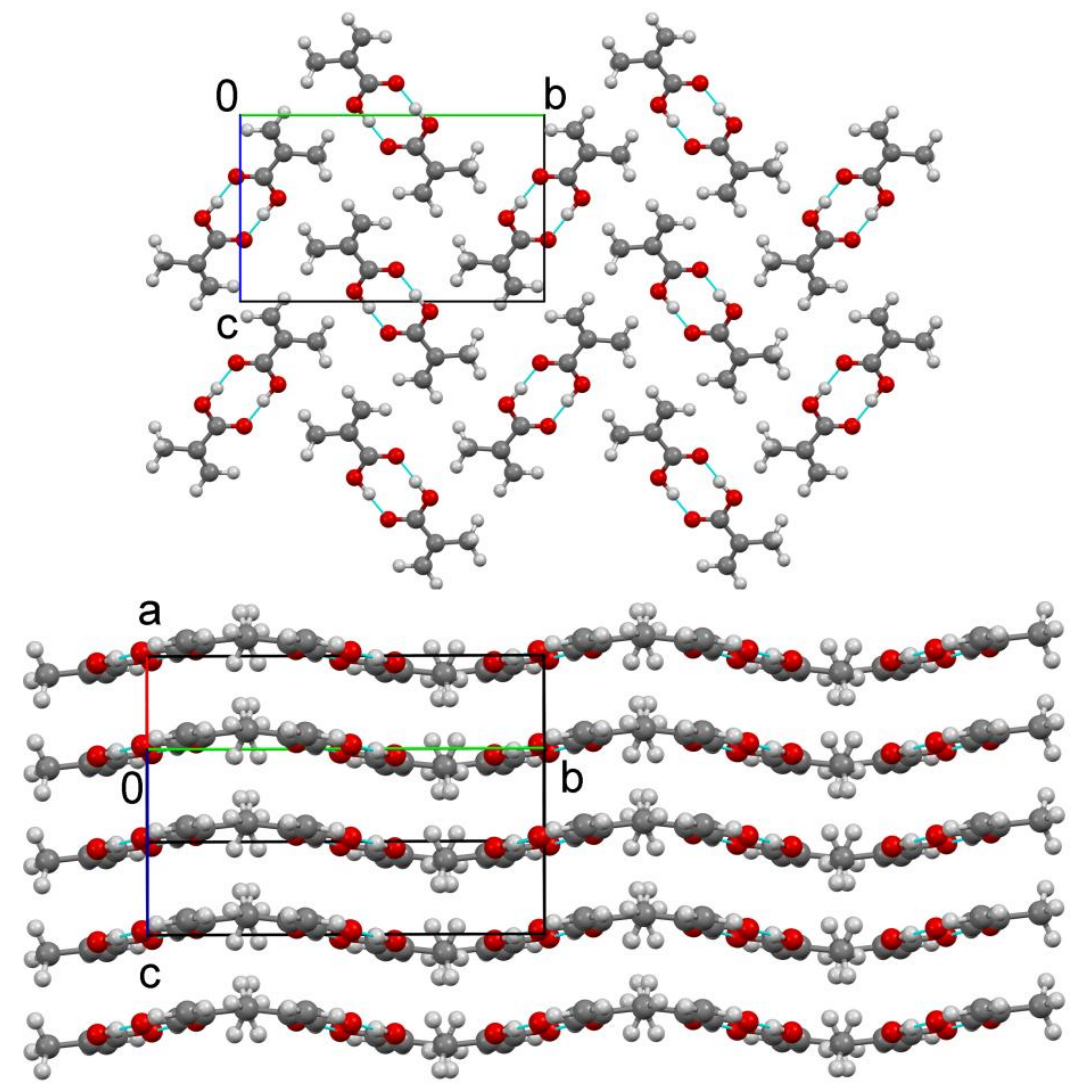

Figure 5: A layer of molecules observed in Phase II methacrylic acid showing the hydrogen bonded dimer (top). A view parallel to the corrugated layers (bottom). Atom colors are assigned as Grey, carbon; Red, oxygen; and White, hydrogen. 


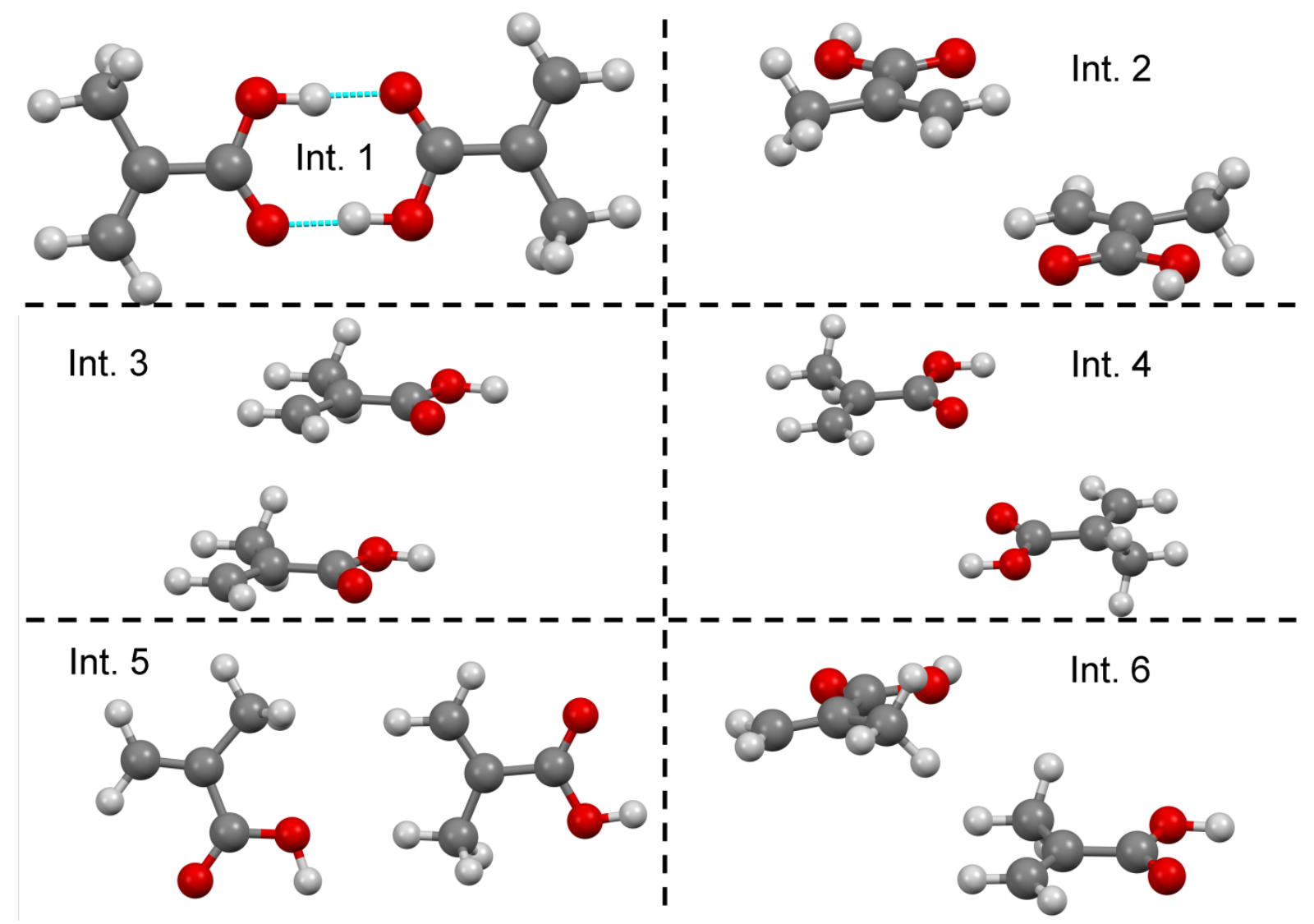

Figure 6: The most energetically favorable interactions in Form II of methacrylic acid with energies $>5$ $\mathrm{kJ} / \mathrm{mol}$. Due to the relative postions of the molecules the interactions have been isolated. Atom colors are assigned as Grey, carbon; Red, oxygen; and White, hydrogen

The lattice parameters, $a, b$ anc $c$, have decreased by $4.1 \%, 1.6 \%, 3 \%$, respectively over $0.9 \mathrm{GPa}$. The arrangement of molecules into a layer parallel to the $b$-axis is the cause of the smaller compression along this direction. The bulk modulus for Phase II was determined to be 7.6(3) GPa with a $\mathrm{V}_{0}$ value of 473.9(16) $\AA^{3}$ and $\mathrm{K}^{\prime}$ fixed at a value of 4 using a $2^{\text {nd }}$-order Birch-Murnaghan equation of state (EOS) in EoSFITGui which is a similar value to that observed for Phase II of acrylic acid. ${ }^{32}$ Over the pressure range there is very little change with respect to intermolecular energies of each of the interactions before a further phase change occurs at 1.2 GPa to a phase that has not been observed before in our previous studies. This is intriguing as the pressure is below that of the single crystal study of Phase II. An explanation for this is that the barrier to conversion from Phase II to Phase III is too large so that once the crystal of Phase II was formed there was a significant kinetic barrier to conversion. This is corroborated by the fact that in our previous work there is no change in the Raman spectra, for either phase, with 
increasing pressure i.e. no I-II or II-III phase transitions were observed. We have observed this in other samples, e.g. glycolide, where powdered material converts to a new phase whilst the single crystal remains in the low pressure phase. ${ }^{33}$

Due to the sample environment and the lack of low angle reflections that are observed the indexing of Phase III proved troublesome. To elucidate the structure of Phase III the same solution was loaded into a DAC and compressed above $1.2 \mathrm{GPa}$ to mimic the neutron experiment. The sample was then left to anneal at approximetely $323 \mathrm{~K}$ for two weeks. Over this time period a crystal of Phase III was grown that provided enough data that could be deconvoluted from the multiple crystallites to solve and refine the new structure. The new phase fits the neutron data very well (Figure ES2). The unit cell parameters are $a=3.6140(7), b=16.420(3), c=6.773(3) \AA, \beta=90.36(3)^{\circ}$, with a volume of $401.91(17) \AA^{3}$. Phase III is observed to be in monoclinic $P 2{ }_{1} / n$ with one molecule in the asymmetric unit. This phase has a herring-bone motif with neighbouring dimers rather than the planar structures observed for the other two phases (Figure 7). The familiar acid dimer has been retained as it is the most favourable interaction observed in the structure $(-50 \mathrm{~kJ} / \mathrm{mol})$. The dimers are stacked parallel to the $a$-axis and the interaction between these dimers that shows two of the most significant interactions in this phase (Int. 2, -15 kJ/mol; and Int.5, $-5 \mathrm{~kJ} / \mathrm{mol}$ ); Int.2 showing a greater coulombic element compared with the rather more dispersive interaction of Int. 5 (Figure 8). These two interactions are equivalent to Int. 3 and 4 in Phase I and II. These dimers are displaced along the length of the interaction with respect to one another compared with Phase II where the dimers are offset diagonally from one another. Over the phase transition (1.31-1.42 GPa) the separation between the dimers (as calcuated using leastsquares plane of atoms) increases from $3.22 \AA$ to $3.28 \AA$ which can be accounted for by the close proximity of the methyl groups. Interactions 3 and 4 are with neighbouring stacks along the $b$ - and $c$-axis respectively. In both of these interactions there is a large dispersion component as these interactions do not contain any hydrogen bonding.

On compression the diffraction pattern rapidly deteriorates which impacts on the quality of the Rietveld refinements of the structures (Figure 4). All the data to 3.9 GPa were able to be refined using the Rietveld method but the last two datasets were not of sufficient quality to be refined and so the unit cell parameters are taken from the Pawley fit of the data. The bulk modulus for this phase calculated using a $2^{\text {nd }}$-order Birch-Murnaghan EOS is $12.4(3) \mathrm{GPa}$ with $\mathrm{V}_{0}$ of $450.2(11) \AA^{3}$ and a $\mathrm{K}$ ' fixed to a value of $4,{ }^{32}$ indicating that it is significantly less compressible than Phase II which is intuitive given the 
observation at higher pressure.

On increasing pressure only two of the interaction show a distinct increase in the energy, the acid dimer (Int. 1) and interaction 5 (above and below). These two terms increase by approximately $10 \mathrm{~kJ} / \mathrm{mol}$ over the course of the compression. Using the geometry optimised values there is a $1.3 \%$ decrease in the hydrogen bond lengths from $1.42-3.89 \mathrm{GPa}(2.581 \AA \mathrm{O} 1 \ldots \mathrm{O} 2$ at $1.42 \mathrm{GPa}$ c.f $2.523 \AA$ at $5.12 \mathrm{GPa})$. Whilst there is an increase in the coulombic and polarisation terms (i.e. more favourable) these increases are negated by the substantial rise in the repulsion term as the molecules are compressed together. Comparing these data with that of the Cambridge Structural Database for the distances between carbonyl carbon atoms across the dimer we see that the distances at $5.12 \mathrm{GPa}$ are at the lower end of the distribution (Figure ES3) which may account for the diffraction becoming worse due to increased repulsion. Other significant changes in intermolecular energies is between the layers; there is a $7 \%$ reduction in the distance between dimers as indicated by Int. $2 \& 5$ (from $3.270 \AA$ at $1.43 \mathrm{GPa}$ to $3.033 \AA$ at 5.12 $\mathrm{GPa}$ ). At $5.12 \mathrm{GPa}$ Int. 5 is the most unfavourable interaction with a final energy value of $+7 \mathrm{~kJ} / \mathrm{mol}$ where there has been a significant increases in the repulsive interaction. To offset this change Int. 2 is decreasing over the course of the compression due to the relatively small contribution of the repulsion term. The overall energy for the structure has increased from $-63 \mathrm{~kJ} / \mathrm{mol}$ to $-40 \mathrm{~kJ} / \mathrm{mol}$ which will be a contributing factor to the deterioration of the diffraction pattern. 

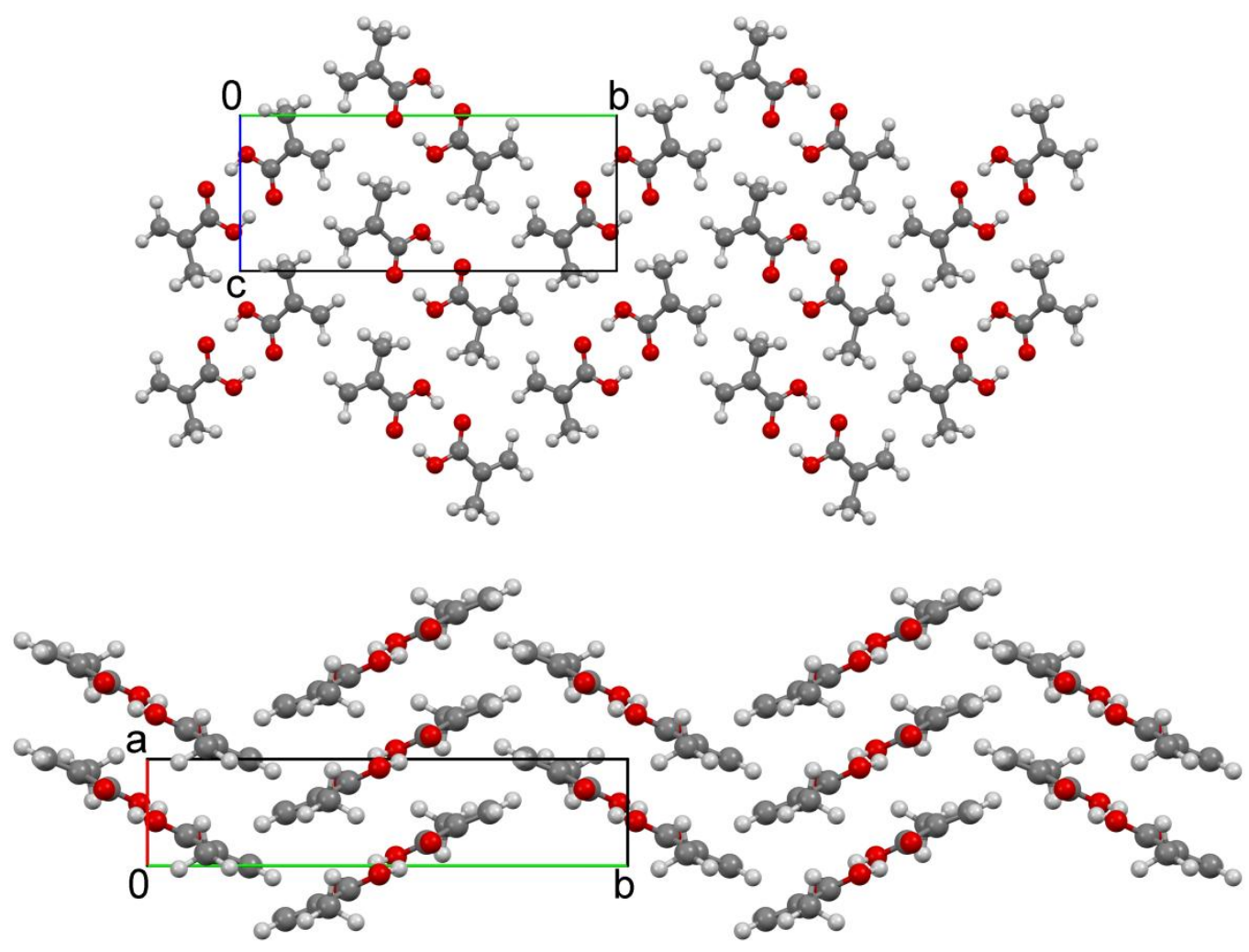

Figure 7: A layer of molecules observed in Phase III methacrylic acid showing the hydrogen bonded dimer (top). A view parallel to the herringbone motif (bottom). Atom colors are assigned as Grey, carbon; Red, oxygen; and White, hydrogen

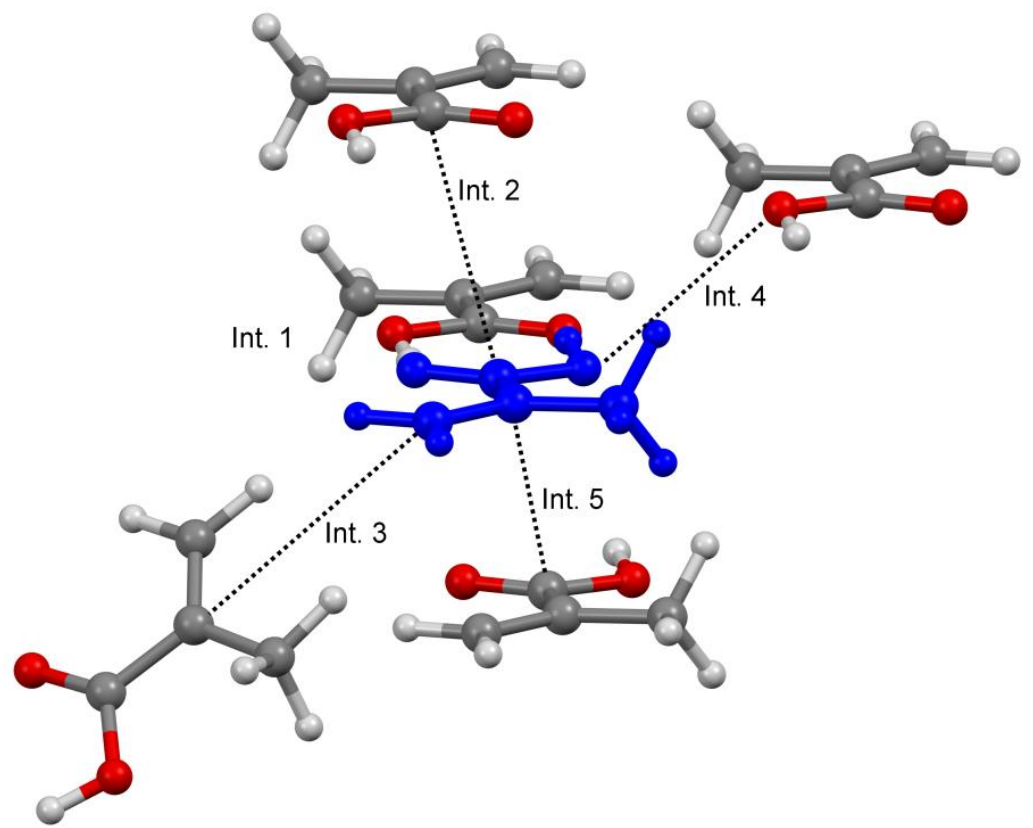

Figure 8: The five most favourable interactions in Phase III. Central molecule is colored blue but the other atoms are present in their own colors; Grey, carbon; Red, oxygen; and White, hydrogen. 
At 5.08 GPa the diffraction pattern had deteriorated and so the behaviour on decompression was explored. The release of pressure is a little less controlled but a number of data points were collected and fitted using the Phase III unit cell parameters; these are designated by hollow green triangles in Figure 3. On decompression Phase III persists to $0.54 \mathrm{GPa}$ before converting directly to Phase I. The Phase III pattern is dominated by two peaks at $\sim 3$ and $\sim 3.4 \AA$ with an undefined number of peaks between them. Only on decompression did the pattern resolve itself so that a number of reflections could be observed between these two main peaks but even with these reflections visible indexing and solution of Phase III was impossible. Phase III was only identified via single crystal X-ray diffraction methods (Figure ES4). Rietveld refinements of methacrylic acid- $d_{6}$ after the compression was possible and showed that the proportion of the pattern attributeable to the methacrylic acid had decreased substantially compared with the patterns at the beginning of the experiment using the fluorite as the internal standard (64\% to $17 \%)$. There was approximately $50 \%$ by weight of material that did not contribute to the diffraction pattern which we assume to be polymer. Previous work on methacrylic acid- $h_{6}$ produced a polymer on decompression from $\sim 8 \mathrm{GPa}$ and so it is likely that this will have occurred in this sample too. ${ }^{9}$

\section{Raman measurements of polymer from methacrylic acid- $h_{6}$ compression}

To equate this work with the literature analysis of the polymer produced from the $-h_{6}$ material Raman spectroscopy was performed. A note worth mentioning at this point is that we have been able to polymerise methacrylic acid by the sole use of pressure; it occurs even if we pressurize without any irradiation (e.g. by laser, X-ray, neutron radiation etc.). In previous literature by Koenig et al. they created polymethacrylic acid via 10 Mrads of radiation using cobalt-60 $\gamma$-radiation which means that pressure is a safer method of synthesis. ${ }^{12}$ The polymer shows a number of similar bands to those found in the literature as shown in Figure 9 with a few notable changes that correspond to free acid despite the polymer being left for a period of time and dried out. At 770 and $805 \mathrm{~cm}^{-1}$ there is a doublet of peaks where there is only one peak found in previous literature with the assignment attributed to the $\mathrm{C} 1-\mathrm{C} 2$ stretch. The $805 \mathrm{~cm}^{-1}$ peak is at a typical range for the free methacrylic acid liquid. Both the ambient and high-pressure polymer have a distinct peak at $\sim 1448 \mathrm{~cm}^{-1}$ which was assigned to the $\mathrm{CH}_{2}$ group but in addition the pressure-induced polymer has a second peak at $1406 \mathrm{~cm}^{-1}\left(\mathrm{CH}_{2}\right.$ deformation) which is 
very close to the peaks observed in the pure compound. Finally, the pressure-induced polymer shows a strong stretch at $\sim 1650 \mathrm{~cm}^{-1}$ which is absent in the ambient produced polymer. This region has is a broad low level stretch that was assigned to the hydrogen bonded carboxylic acid. Whilst the pressure-induced sample does show shoulders that may be equivalent this region is dominated by the $\sim 1650 \mathrm{~cm}^{-1}$ stretch which was assigned by Badawi et al. to the $\mathrm{C}=\mathrm{C}$ stretch or $\mathrm{CH} 2$ bend from the pure unreacted molecule. ${ }^{30}$ There are two potential reasons for the appearance of these sharper peaks: i) the polymer itself is of low molecular weight and therefore has a number of free methylene groups that contribute to these vibration; or ii) that there may be some residual acid encapsulated within the polymer. We suspect due to the sharpness and wavenumber of the peaks that there must be some residual liquid encapsulated in the polymer. From the spectroscopic analysis there does not seem to be much difference between our polymer and the syndiotactic polymethacrylic acid observed by Koenig et al. ${ }^{12}$ The most probable route of polymerisation is via Interaction 5. The spatial arrangement of the molecules aligns the 'head' of the ethylene moeity with the 'tail' of the nearest neighbour at a value of $\sim 3.2 \AA$; this distance being cited as a viable distance for solid-state polymeristaion. ${ }^{4-5,10}$ The significant increase in the repulsive term over the course of the compression indicating the instability of the molecular interaction. If this is the route then there must be a molecular rearrangement through bond rotation to arrive at the syndiotactic arrangement. 


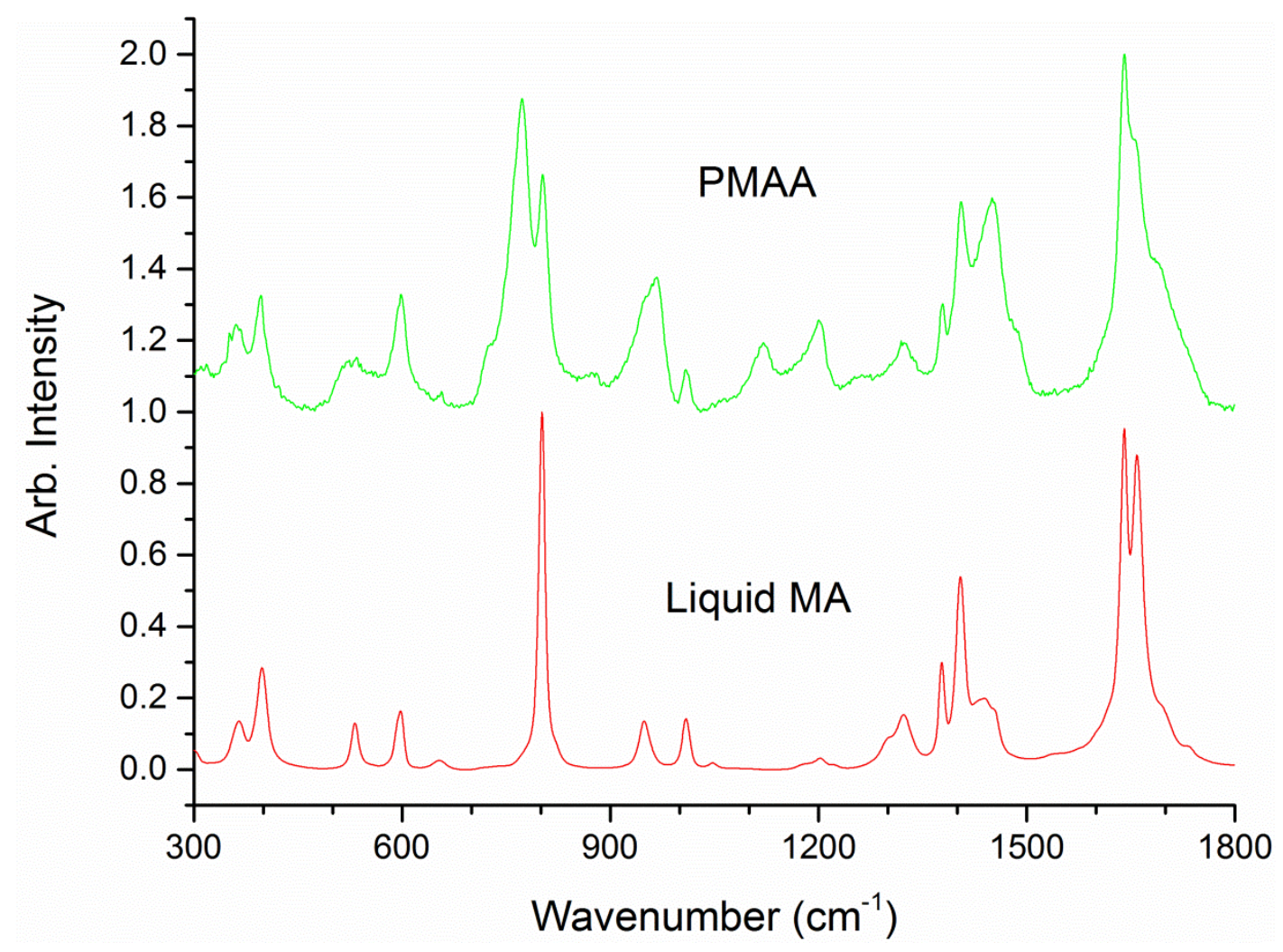

Figure 9: A selected portion of the Raman spectra for the pressure synthesised polymer (PMAA, above) and pure methacrylic acid (MA, bottom).

\section{Conclusions}

We have confirmed that the methacrylic acid- $d_{6}$ undergoes two polymorphic transitions at 0.39 and $1.20 \mathrm{GPa}$. The first of these transitions is between two known phases but the second transition is to a new polymorphic form that has been characterised as part of this study. Diffraction from Phase III deteriorates rapidly but can still be refined as a molecular form. Phase III persists on decompression to $0.54 \mathrm{GPa}$ before transforming to Phase I. The proportion of crystalline methacrylic acid as decreased substantially from $64 \mathrm{wt} \%$ to $17 \mathrm{wt} \%$ with approximately $50 \%$ weight that is not giving rise to diffraction; we believe this to be the polymer product. Pressure has been shown to be a novel route to the production of polymers where other routes have used significant doses of $\gamma$-radiation.

\section{Acknowledgements}

The authors would like to thank Craig Bull for technical assistance in the preparation of this manuscript. Experiments at the ISIS Pulsed Neutron and Muon Source were supported by a 
beamtime allocation from the Science and Technology Facilities Council (RB1110228 \& RB1310267). The authors would also like to thank Ian Hutchison and Amit Delori for their assistance on beamtime and The Leverhulme Trust for funding RPG-2012-598.

\section{Supporting Information Available}

Information relating to the experimental methods of Raman, IR, DSC and Cambridge Structural Database searches can be found in the SI. Figures of the powder neutron diffraction, IR and DSC are shown. Tabulated information of the full crystallographic information and Pixel energies are also presented. This information is available free of charge via the Internet at http://pubs.acs.org.

\section{References}

1. Bini, R.; Ceppatelli, M.; Citroni, M.; Schettino, V. From Simple to Complex and Backwards. Chemical Reactions under Very High Pressure. Chem Phys 2012, 398, 262-268.

2.Chelazzi, D.; Ceppatelli, M.; Santoro, M.; Bini, R.; Schettino, V. Pressure-Induced Polymerization in Solid Ethylene. J Phys Chem B 2005, 109 (46), 21658-21663.

3. Ciabini, L.; Santoro, M.; Bini, R.; Schettino, V. High Pressure Reactivity of Solid Benzene Probed by Infrared Spectroscopy. J. Chem. Phys. 2002, 116 (7), 2928-2935.

4. Jin, H. J.; Plonka, A. M.; Parise, J. B.; Goroff, N. S. Pressure Induced Topochemical Polymerization of Diiodobutadiyne: a Single-Crystal-to-Single-Crystal Transformation. Crystengcomm 2013, 15 (16), 3106-3110.

5. Wilhelm, C.; Boyd, S. A.; Chawda, S.; Fowler, F. W.; Goroff, N. S.; Halada, G. P.; Grey, C. P.; Lauher, J. W.; Luo, L.; Martin, C. D., et al. Pressure-Induced Polymerization of Diiodobutadiyne in Assembled Cocrystals. J Am Chem Soc 2008, 130 (13), 4415-4420.

6. Santoro, M.; Gorelli, F. A.; Bini, R.; Ruocco, G.; Scandolo, S.; Crichton, W. A. Amorphous SilicaLike Carbon Dioxide. Nature 2006, 441 (7095), 857-860.

7. Ceppatelli, M.; Frediani, M.; Bini, R. High-Pressure Reactivity of L,L-Lactide. J Phys Chem B 2011, 115 (10), 2173-2184.

8. Murli, C.; Mishra, A. K.; Thomas, S.; Sharma, S. M. Ring-Opening Polymerization in Carnosine under Pressure. J Phys Chem B 2012, 116 (15), 4671-4676.

9. Oswald, I. D. H.; Urquhart, A. J. Polymorphism and Polymerisation of Acrylic and Methacrylic Acid at High Pressure. Crystengcomm 2011, 13 (14), 4503-4507.

10. Johnston, B. F.; Marshall, W. G.; Parsons, S.; Urquhart, A. J.; Oswald, I. D. H. Investigation of Acrylic Acid at High Pressure Using Neutron Diffraction. J Phys Chem B 2014, 118 (14), 40444051.

11. Oswald, I. D. H.; Crichton, W. A. Structural Similarities of 2-chlorophenol and 2methylphenol. Crystengcomm 2009, 11 (3), 463-469.

12. Koenig, J. L.; Angood, A. C.; Semen, J.; Lando, J. B. Laser-excited Raman Studies of the Conformational Transition of Syndiotactic Polymethacrylic Acid in Water. J Am Chem Soc 1969, 91 (26), 7250-7254.

13. ISIS_Dedicated_Facility_for_High_Pressure_Diffraction ISIS 96-ISIS Facility Annual Report 1995-96; Rutherford Appleton Laboratory: 1996; pp 61-62.

14. ISIS_PEARL_Pressure_and_Engineering_Research_Line ISIS 97 - ISIS Facility Annual Report 1996-97; Rutherford Appleton Laboratory: 1997; pp 28-29.

15. Besson, J. M.; Nelmes, R. J.; Hamel, G.; Loveday, J. S.; Weill, G.; Hull, S. Neutron Powder Diffraction above 10-Gpa. Physica B 1992, 180, 907-910.

16. Funnell, N. P.; Marshall, W. G.; Parsons, S. Alanine at $13.6 \mathrm{GPa}$ and its Pressure-Induced Amorphisation at $15 \mathrm{GPa}$. Crystengcomm 2011, 13 (19), 5841-5848.

17. Marshall, W. G.; Francis, D. J. Attainment of Near-Hydrostatic Compression Conditions using the Paris-Edinburgh Cell. J Appl Crystallogr 2002, 35, 122-125. 
18. Angel, R. J. The High-Pressure, High-Temperature Equation of State of Calcium Fluoride, $\mathrm{CaF}_{2}$. J. Phys.-Condes. Matter 1993, 5 (11), L141-L144.

19. Coelho, A. TOPAS - Academic: General Profile and Structure Analysis Software for Powder Diffraction Data, Version 5; 2012.

20. Funnell, N. P.; Dawson, A.; Marshall, W. G.; Parsons, S. Destabilisation of Hydrogen Bonding and the Phase Stability of Aniline at High Pressure. Crystengcomm 2013, 15 (6), 1047 1060.

21. Delley, B. An All-Electron Numerical-Method for Solving the Local Density Functional for Polyatomic-Molecules. J Chem Phys 1990, 92 (1), 508-517.

22. Accelrys_Software_Inc, In Materials Studio Release Notes, Release 6, San Diego, 2011.

23. Perdew, J. P.; Chevary, J. A.; Vosko, S. H.; Jackson, K. A.; Pederson, M. R.; Singh, D. J.; Fiolhais, C. Atoms, Molecules, Soilds, and Surfaces- Applications of the Generalized Gradient Approximation for Exchange and Correlation. Phys Rev B Condens Matter 1992, 46 (11), 66716687.

24. Tkatchenko, A.; Scheffler, M. Accurate Molecular Van Der Waals Interactions from Ground-State Electron Density and Free-Atom Reference Data. Phys Rev Lett 2009, 102 (7), 073005.

25. Monkhorst, H. J.; Pack, J. D. Special Points for Brillouin-Zone Integrations. Phys Rev B 1976, 13 (12), 5188-5192.

26. Gavezzotti, A. Non-Conventional Bonding between Organic Molecules. The 'Halogen Bond' in Crystalline Systems. Mol Phys 2008, 106 (12-13), 1473-1485.

27. Gavezzotti, A. Calculation of Intermolecular Interaction Energies by Direct Numerical Integration over Electron Densities. 2. An Improved Polarization Model and the Evaluation of Dispersion and Repulsion Energies. J Phys Chem B 2003, 107 (10), 2344-2353.

28. Frisch, M. J.; Trucks, G. W.; Schlegel, H. B.; Scuseria, G. E.; Robb, M. A.; Cheeseman, J. R.; Scalmani, G.; Barone, V.; Mennucci, B.; Petersson, G. A., et al., Gaussian 09, Revision B.01. Wallingford CT, 2009.

29. Macrae, C. F.; Bruno, I. J.; Chisholm, J. A.; Edgington, P. R.; McCabe, P.; Pidcock, E.; Rodriguez-Monge, L.; Taylor, R.; van de Streek, J.; Wood, P. A. Mercury CSD 2.0 - New Features for the Visualization and Investigation of Crystal Structures. J Appl Crystallogr 2008, 41 (2), 466470.

30. Badawi, H. M.; Al-Khaldi, M. A. A.; Al-Abbad, S. S. A.; Al-Sunaidi, Z. H. A. Rotational Barriers in Monomeric $\mathrm{CH} 2 \mathrm{CX}-\mathrm{COOH}$ and $\mathrm{CH} 2 \mathrm{CX}-\mathrm{CONH} 2(\mathrm{X}$ is $\mathrm{H}$ or $\mathrm{CH} 3$ ) and Vibrational Analysis of Methacrylic Acid and Methacrylamide. Spectrochim. Acta, Part A 2007, 68 (3), 432-442. 31. Allen, F. H.; Baalham, C. A.; Lommerse, J. P. M.; Raithby, P. R. Carbonyl-Carbonyl Interactions can be Competitive with Hydrogen Bonds. Acta Crystallogr. B 1998, 54, 320-329.

32. Angel Ross, J.; Alvaro, M.; Gonzalez-Platas, J., EosFit7c and a Fortran Module (Library) for Equation of State Calculations. In Zeitschrift für Kristallographie - Crystalline Materials, 2014; Vol. 229, p 405.

33. Hutchison, I. B.; Delori, A.; Wang, X.; Kamenev, K. V.; Urquhart, A. J.; Oswa Id, I. D. H. Polymorphism of a Polymer Precursor: Metastable Glycolide Polymorph Recovered via Large Scale High-Pressure Experiments. Crystengcomm 2015, 17 (8), 1778-1782. 
Keywords: Methacrylic acid, Pressure, Reconstructive phase transition, Polymerisation, Pixel, 
TOC

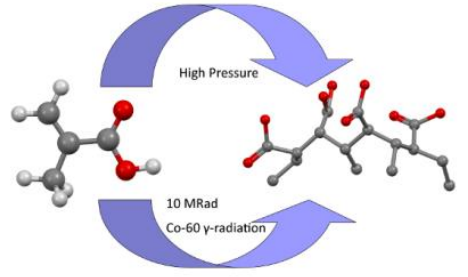

